\title{
Up-regulation of long noncoding RNA MALAT1 contributes to proliferation and metastasis in esophageal squamous cell carcinoma
}

\author{
Liwen $\mathrm{Hu}^{1}$, Yuanyuan $\mathrm{Wu}^{2}$, Deli Tan ${ }^{1}$, Hui Meng ${ }^{2}$, Kai Wang ${ }^{2}$, Yun Bai ${ }^{2^{*}}$ and Kang Yang ${ }^{1 *}$
}

\begin{abstract}
Background: Metastasis Associated Lung Adenocarcinoma Transcript 1 (MALAT1) has been demonstrated to be an important player in various human malignancies; it is thought to promote tumor growth by cell cycle regulating. However, the roles of MALAT1 in esophageal squamous cell carcinoma(ESCC), and the mechanisms involved in cell cycle regulation remain poorly understood. Moreover, the factors contributing to its up-regulation in tumor tissues are still largely unclear.

Methods: Expression of MALAT1 was determined from cell lines and clinical samples by qRT-PCR. The effects of MALAT1 knockdown on cell proliferation, cell cycle, apoptosis, migration, and invasion were evaluated by in vitro and in vivo assays. The potential protein expression changes were investigated by Western-blotting. The methylation status of the CPG island in the MALAT1 promoter was explored by bisulfite sequencing, while the copy numbers in tumor tissues and blood samples were detected by a well-established AccuCopy ${ }^{\text {TM }}$ method.

Results: MALAT1 was over-expressed in $46.3 \%$ of ESCC tissues, mostly in the high-stage tumor samples. Enhanced MALAT1 expression levels were positively correlated with clinical stages, primary tumor size, and lymph node metastasis. Inhibition of MALAT1 suppressed tumor proliferation in vitro and in vivo, as well as the migratory and invasive capacity. MALAT1 depletion also induced G2/M phase arrest and increased the percentage of apoptotic cells. Western-blotting results implicated that the ATM-CHK2 pathway which is associated with G2/M arrest was phosphorylated by MALAT1 knockdown. No effects of CpG island methylation status on MALAT1 expression were found, whereas amplification of MALAT1 was found in $22.2 \%$ of tumor tissues, which correlated significantly with its over-expression. However, neither association between tissue copy number amplification and germline copy number variation, nor correlation between germline copy number variation and ESCC risk were identified in the case-control study.
\end{abstract}

Conclusions: Our data suggest that MALAT1 serves as an oncogene in ESCC, and it regulates ESCC growth by modifying the ATM-CHK2 pathway. Moreover, amplification of MALAT1 in tumor tissues may play an important role for its up-regulation, and it seems that the gene amplification in tumor tissues emerges during ESCC progression, but is not derived from germline origins.

Keywords: Long noncoding RNA, MALAT1, Esophageal cancer, Copy number, Cell cycle arrest

\footnotetext{
* Correspondence: yunbai@tmmu.edu.cn; yangkangxwk@163.com

${ }^{2}$ Department of Medical Genetics, College of Basic Medical Science, Third Military Medical University, Gaotanyan St., Shapingba District, Chongqing, People's Republic of China

'Department of Cardiothoracic Surgery, Southwest Hospital, Third Military Medical University, Gaotanyan St., Shapingba District, Chongqing, People's Republic of China
} 


\section{Background}

Esophageal cancer is one of the leading aggressive malignancies worldwide and ranks as one of the top five deadliest cancers in China, while squamous cell carcinoma accounts for the most prevalent histopathologic type [1]. Despite the recent advances in ESCC treatment, the prognosis of ESCC is still unfavorable [2,3], making it essential for uncovering the underlying mechanisms of ESCC for therapy improvement. The emerging roles of lncRNAs in human tumorgenesis may hold great promise for understanding the carcinogenesis of ESCC [4].

Long noncoding RNA (lncRNA) is defined as a group of RNAs (>200 nt) with limited or no coding capacity due to a lack of an intact open reading frame, but participates in most vital biological activities. LncRNAs can affect gene expression via multiple fashions, including chromatin reprogramming [5], X chromosome inactivation [6], microRNA sponging [7], and so on. A growing amount of literature has linked deregulation of IncRNAs with diverse human cancers, such as the well-characterized HOTAIR, MEG3, GAS5, H19, and MALAT1 [8].

Metastasis Associated Lung Adencarcinoma Transcript 1 (MALAT1), mapped to human chromosome 11q13, was originally identified as a prognostic marker for metastasis and patient survival in NSCLC [9]. Following studies have documented that MALAT1 was aberrantly up-regulated in multiple cancerous tissues and conferred proliferative and metastatic phenotypes to tumor cells [10-12]. It promotes tumor growth and metastasis through different mechanisms, including by recruiting SR family proteins [13], binding to the active regions of chromosome [14], and regulating alternative splicing of oncogenic mRNAs [15], depending on tissue contexts. The role that it plays in ESCC is of interest to our research group. Kuo et al. reported that exogenous over-expression of tumor suppressor gene sox17 impaired ESCC growth and mobility, with a synchronous decreased MALAT1 level [16], which indirectly implicated that MALAT1 may serve as an oncogene in ESCC.

There is a lot of evidence suggesting that MALAT1 may be involved in cell cycle regulation, which may contribute to uncontrolled tumor growth. Its role in $\mathrm{G} 2 / \mathrm{M}$ regulation has been specifically emphasized $[15,17,18]$, but its detailed mechanism is still controversial. Tripathi et al. reported that MALAT1 depletion leads to an increased level of $\gamma \mathrm{H} 2 \mathrm{AX}$ (a DNA damage indicator) and accumulated G2/M population, indicating a critical involvement of MALAT1 in genome stability and cell cycle checkpoint. To our knowledge, the tumor genomes are under frequent DNA damage events and spontaneous genetic mutation, which could activate the cell cycle checkpoints, represented by the ATM-CHK2 pathway. The activated checkpoint may prevent tumors from growing too quickly by inducing cell cycle arrest. Inactivation of this checkpoint may release cells from cycle arrest, and results in accelerating tumor growth. In consideration of the role of MALAT1 in cell cycle progression and genome stability maintenance, we speculated that MALAT1 could promote ESCC proliferation by regulating the ATM-CHK2 pathway, which is associated with DNA damage response and the $\mathrm{G} 2 / \mathrm{M}$ transition.

Moreover, the factors that lead to MALAT1 overexpression in tumor tissues remain largely unknown, previous studies have found that the expression level of MALAT1 was controlled by methylation of histone H3 [19], transcriptional factors [16,20], and microRNAs [21,22]; however, evidence to explain its over-expression in diverse tumor tissues is lacking.

To better understand its roles in ESCC and the factors leading to its up-regulation, we investigated the clinical significance of MALAT1 on ESCC and confirmed its biological functions by in vitro and in vivo assays. We also checked the ATM-CHK2 pathway, which is involved in DNA damage response and G2/M arrest, to unravel the mechanisms by which MALAT1 regulates cell cycle progression and promotes ESCC growth. To explore the factors contributing to its upregulation, we sequenced the $\mathrm{CPG}$ island located at its promoter, and detected the copy number alterations in tumor tissues. Finally, we determined whether the MALAT1 amplification in tumor tissues was derived from germline origins. We also evaluated the possibility that the germline copy number variation (CNV) of MALAT1 be used as an indicator of ESCC risk for Chinese Han people in a case-control study.

Our results showed that MALAT1 was upregulated mostly in late-stage tumor tissues, indicating that it mainly functions in the advanced stages of ESCC but not tumor initialization. Knockdown of MALAT1 suppressed proliferation and metastasis of ESCC cells, leading to $\mathrm{G} 2 / \mathrm{M}$ arrest and an increased apoptosis ratio. MALAT1 depletion activated the ATM-CHK2 pathway, which should be responsible for $\mathrm{G} 2 / \mathrm{M}$ arrest. Our results also revealed a negative association between MALAT1 expression and ATM-CHK2 pathway phosphorylation in tumor tissues, suggesting that up-regulation of MALAT1 may promote ESCC growth by dephosphorylation of the ATM-CHK2 pathway, which may loose the cell cycle arrest. We also found that amplification of MALAT1 in tumor tissues may partially contribute to its overexpression, but the genomic amplification in somatic tissues should be a complex event, instead of being derived from a germline source. In addition, the casecontrol study results indicated that the germline CNV of MALAT1 should not be treated as an indicator for ESCC susceptibility. 


\section{Materials and methods}

\section{Tissue samples and cell lines}

ESCC and corresponding normal esophageal epithelial tissues were obtained from 54 patients who underwent surgery resection during 2007-2012 at Southwest Hospital, Chongqing, China. No patient recruited in the study received radio- or chemo-therapy prior to surgery. Clinical information was collected from medical records. All specimens were snap-frozen and stored at $-80^{\circ} \mathrm{C}$ until use. The study was approved by the Research Ethics Committee of the Third Military Medical University, Chongqing, China. Written informed consent for biological research was obtained from all participants.

Four esophageal squamous cell carcinoma cell lines (EC109, EC9706, KYSE150, and KYSE450) were obtained from the Cell Bank of the Chinese Academy of Sciences (Shanghai, China) and Cancer Institute and Hospital, Chinese Academy of Medical Sciences (Beijing, China). A normal esophageal epithelial cell (Het-1A) was purchased from Jenniobio Biotechnology (Guangzhou, China). All cells were cultured in RPMI-1640 medium (Hyclone, USA) supplemented with $10 \%$ fetal bovine serum (10\% FBS), and maintained in a humidified incubator at $37^{\circ} \mathrm{C}$ with $5 \% \mathrm{CO}_{2}$.

\section{Case-control study population}

A total of 201 cases diagnosed with ESCC and 193 ethnically-matched healthy controls were recruited from Southwest Hospital. All subjects were genetically unrelated ethnic Han-Chinese from southwest China. The protocol and consent form were approved by the Research Ethics Committee of the Third Military Medical University. All participants provided informed consent prior to enrollment.

Distribution of demographic characteristics are listed in Additional file 1: Table S1.

\section{RNA extraction and qRT-PCR}

Total RNA was isolated from tissues and cell cultures with Trizol reagent (Takara, Japan) according to the standard protocol. The cDNA was synthesized from a total of 200 ng RNA using the PrimeScript RT reagent Kit (Takara, Japan) and amplified by quantitative real-time PCR with SYBR Green Kit (Takara, Japan) on Illumina $\mathrm{ECO}^{\mathrm{TM}}$ (Illumina, USA). GAPDH was used as the internal reference, and the relative expression level of MALAT1 was normalized to GAPDH.

Primers for MALAT1 and GAPDH are listed in Additional file 2: Table S2.

\section{DNA extraction}

DNA was extracted from tumor tissues and blood samples using the $\mathrm{Wizard}^{\oplus}$ genomic DNA extraction Kit (Promega, USA) according to the standard procedure, the extracted genomic DNA was dissolved in DNA rehydration solution and stored at $-20^{\circ} \mathrm{C}$ until use.

\section{Small interfering RNA}

Two small interfering RNAs (siRNAs) against MALAT1 (si-MALAT1) at different sites and one negative control (si-NC) with no definite target were employed and synthesized by GenePharma (Shanghai, China). Cells were seeded on six-well plates at a density of $3 \times 10^{5} /$ well overnight, and then transfected with siRNA or the negative control at a final concentration of $100 \mathrm{nM}$ using Lipofectamine 2000 (Invitrogen, USA). The interfering efficiency was determined by qRT-PCR 48 h after transfection, and the siRNAs with silencing efficacy of more than $70 \%$ were selected for further experiments.

Sequences of siRNAs and negative control are provided in Additional file 3: Table S3.

\section{Cell proliferation assay}

Cells seeded on 96-well plates (5000/well) were transfected with siRNAs or NC, and the cell proliferation assays were conducted every 24 h using cell counting kit8 (Djingo, Japan) as the manufacturer's protocol. The number of viable cells was quantified by the absorbance of reduced WST-8 at $450 \mathrm{~nm}$ at indicated time points.

For colony forming experiments, cells (EC109 and EC9706) transfected with siRNAs or NC were plated on six-well plates at a density of 500/well, maintained in RPMI-1640 medium containing 10\% FBS for 12 days. Medium was replaced every 3 days. Colonies were fixed with methanol twelve days later and stained with $0.1 \%$ crystal violet (Sigma, USA). Visible colonies were photographed and counted manually.

\section{Flow-cytometric analysis of apoptosis and cell cycle}

Cells seeded on six-well plates were harvested $72 \mathrm{~h}$ post transfection by trypsin and collected by centrifugation. Cell pellets were washed with cold PBS, and then fixed in $70 \%$ ethanol at $4^{\circ} \mathrm{C}$ overnight. After fixation, the cells were washed and resuspended in PBS, and then incubated with ribonuclease at $37^{\circ} \mathrm{C}$ for 30 minutes, next stained with propidium iodide (Beyotime, China) in the dark at $4^{\circ} \mathrm{C}$ for 30 minutes, and finally analyzed by a flow cytometer FACSCalibur (BD Bioscience, USA) and Modlfit software (Verity Software House, USA).

For apoptosis analysis, the trypsinized and PBS washed cells were stained with FITC-Annexin V and propidium iodide (Beyotime, China). Cells were analyzed by FACSCalibur and Flowjo software (BD Bioscience, USA). Cells were discriminated into viable cells, dead cells, early apoptotic and late apoptotic cells, relative ratios of the early and late apoptotic cells were compared to the control group. 


\section{Transwell assay}

Cell migration and invasion assays were performed using Costar chambers containing transwell inserts with a pore size of $8 \mu \mathrm{m}$ (Corning Incorporated, USA). The upper chambers were either non-coated for migration or coated with Matrigel (Invitrogen, USA) for the invasion assay. Cells $\left(5 \times 10^{4}\right)$ transfected with siRNAs or NC were suspended in $200 \mu \mathrm{l}$ of serum-free medium and seeded in the upper chamber, while the bottom chamber contained medium mixed with $20 \%$ FBS. Thirty-six hours after incubation, cells in the upper chamber were removed by cotton swap, whereas the cells that had migrated or invaded through the membrane were fixed with methanol and stained with $0.1 \%$ crystal violet, imaged, and counted under an inverted microscope in five random fields (Olympus, Japan).

\section{Tumor xenografts in nude mice}

Four-week athymic female BALB/c mice were housed and maintained in laminar airflow chambers under specific pathogen-free conditions. Lentiviral vectors for si-MALAT1 and NC were separately constructed by Genepharm, and the stably transfected EC109 cell lines were established. The transfected EC109 cells were suspended in PBS at a final concentration of $1 \times 10^{8}$ cells $/ \mathrm{ml}$ separately, a volume of $0.1 \mathrm{ml}$ of suspended cells was subcutaneously injected into a single side of the posterior flank of each mouse ( $n=5$ /group). At 21 days post injection, the mice were sacrificed by cervical dislocation in diethyl ether anesthesia, and the tumors were dissected and weighed. The experimental protocols were approved by the committee on animal experimentation of the Third Military Medical University.

\section{Western blotting}

Cells transfected with siRNAs or NC were lysed using protein extraction reagent RIPA (Beyotime, China) $72 \mathrm{~h}$ post transfection, supplemented with protease inhibitors PMSF (Roche, Switzerland). ESCC tissues were ground in homogenizers and proteins were extracted following the manufacturer's instructions (Beyotime, China). Half of the ESCC tissues showed MALAT1 over-expression and were higher than the mean expression level in ESCC, while the other half didn't. Equal amounts of protein extractions were separated by $10 \%$ SDS-polyacrylamide gel electrophoresis (SDS-PAGE), then transferred to polyvinylidene diflvoride membranes (Sigma, USA). The membranes were blocked for $1 \mathrm{~h}$ at room temperature, and subsequently incubated with specific primary antibodies for $12 \mathrm{~h}$. Then the membranes were washed three times and incubated with the horseradish peroxidase (HRP)-conjugated secondary antibodies (anti-rabbit) for one hour. ECL chromogenic substrate was used to visualize the bands. GAPDH was used as the control. Antibodies were all purchased from Abcam (USA).

\section{Methylation status analysis of CpG island}

Genomic DNA prepared from ten pairs of ESCC and normal tissues was modified by sodium bisulfite using the EZ DNA Methylation Kit (Zymo Research, USA), and then amplified by PCR. PCR-amplified products were ligated to pMD18-T vectors (TAKARA, Japan) and transformed into E.coli $\mathrm{DH} 5 \alpha$ cells. The $\mathrm{LB}^{\mathrm{amp}+}$ agar/X-gal/IPTG plates were used to select the qualified colonies. Ten colonies for each sample were picked out and then cultured in $\mathrm{LB}^{\mathrm{amp}+}$ medium overnight separately, and subsequently obtained plasmids were subjected to sequencing.

Primers for CpG island amplification are available in Additional file 2: Table S2.

\section{Treatment of ESCC cells with demethylase 5-aza-cdR}

EC109 and EC9706 cells $\left(3 \times 10^{5}\right)$ were plated on sixwell plates and grown overnight. After $24 \mathrm{~h}$, the media was removed and replaced with fresh media containing $0,5,10 \mu \mathrm{M}$ of demethylase 5-aza-CdR (Sigma, USA). The cells treated with 5-aza-CdR were harvested $48 \mathrm{~h}$ post treatment and subjected to RNA extraction to determine the MALAT1 expression level. To ensure that the demethylation by 5 -aza-CdR was actually occurring, the CDH1 gene which was reported to be regulated by CpG methylation in ESCC [23,24]. Primers for CDH1 are listed in Additional file 2: Table S2.

\section{Copy number genotyping}

Copy number of MALAT1 in tumor tissues and blood samples were interrogated by a custom-by-design AccuCopy ${ }^{\mathrm{TM}}$ kit (Cat\#: CN0105, Genesky Biotechnologies Inc, Shanghai, China), which was developed based on a patented technology from Genesky Biotechnologies Inc. The basic principal of this method has been described previously [25], which was based on a multiplex fluorescence competitive PCR, in brief. To ensure the accuracy of copy number calculations, four reference segments were utilized for normalization. A probe targeted to MALAT1 (1500-1813 bp) was designed for MALAT1 copy number detecting. The primers for the MALAT1 probe and reference segments are provided in Additional file 2: Table S2. Our data were produced mainly according to the manufacturer's manual.

\section{Statistical analysis}

Each biological experiment was performed in triplicate and repeated three times. Data are shown as mean \pm standard deviation (SD). Statistical significance was tested by Student's $t$-test (two-tailed), Chi-square test and oneway ANOVA analysis as appropriate. An unconditional logistic regression model was used to assess the associations between MALAT1 copy number genotypes and ESCC risk 
with or without adjustment by age, gender, and smoking status. All statistical analyses were performed on SPSS 16.0. $P<0.05$ was considered as significant.

\section{Results}

MALAT1 expression was up-regulated predominantly in advanced-stage ESCC tissues

qRT-PCR was used to detect MALAT1 expression levels in cell lines and clinical samples, which were normalized to GAPDH. All the four ESCC cell lines expressed higher MALAT1 than the normal cell line, especially in EC109 and KYSE150 (Figure 1A).

The results from tissues showed that MALAT1 was up-regulated in $46.3 \%$ (25/54, Figure 1B) of ESCC tissues (cancer/normal ratio $>1.5$ ), with the mean expression level of 4.53 in tumors and 3.02 in their counterparts $(p=0.02$, Figure 1C). Furthermore, correlation analysis of MALAT1 expression with clinicopathological parameters revealed

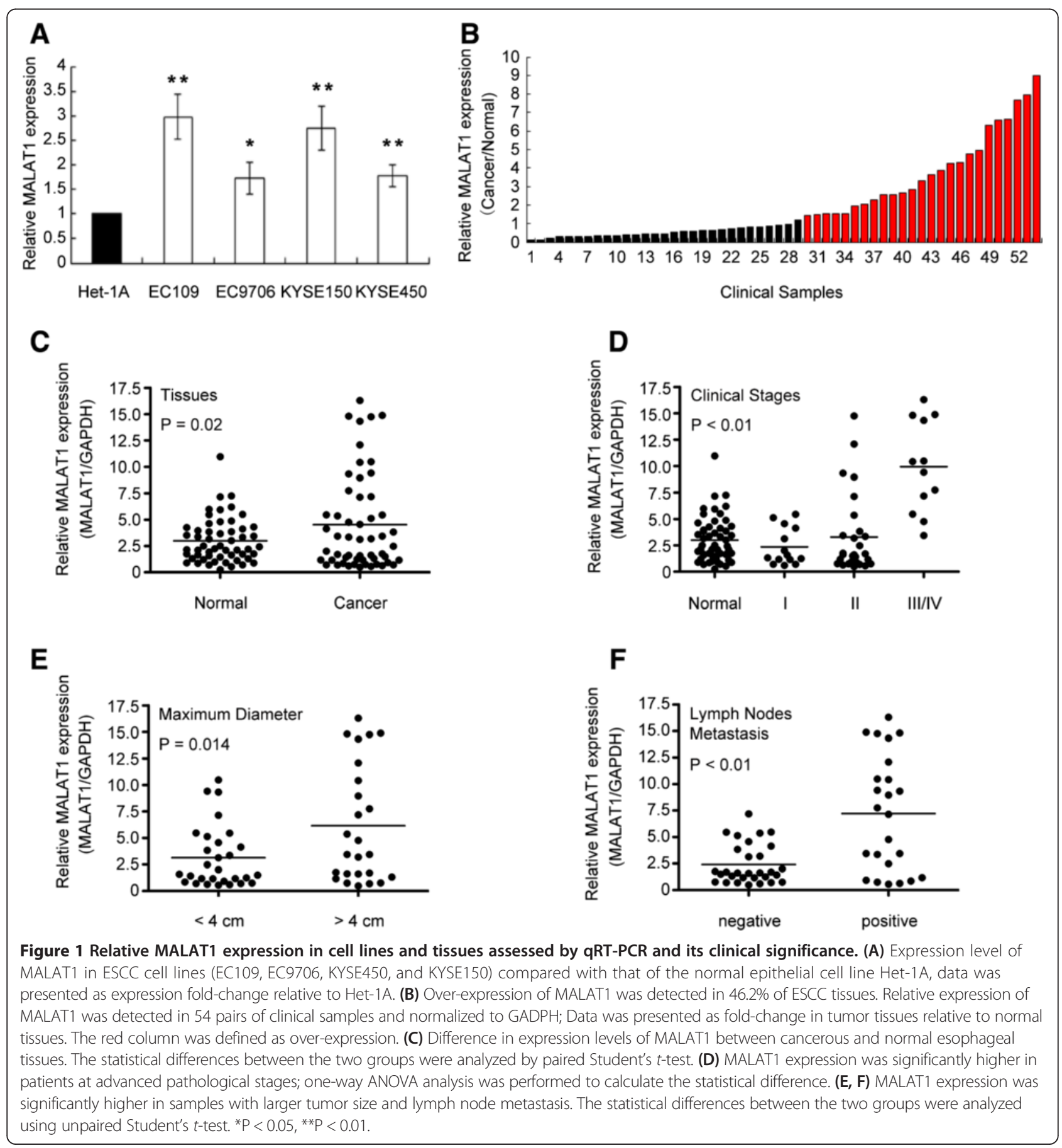


that MALAT1 was predominantly up-regulated in latestage but not early-stage tumor tissues (Figure 1D), which indicated that MALAT1 may play its oncogenic role mostly in ESCC with advancing progression but not in the initial phase. Additionally, we found a positive association of MALAT1 expression with tumor size and lymph nodes metastasis (Figure $1 \mathrm{E}$ and $\mathrm{F}$ ).

We next classified the 54 ESCC patients into two groups according to MALAT1 expression level (over-expression or not). Clinical pathologic factors were compared between the two groups (Table 1). The over-expression group was correlated with larger tumor size $(\mathrm{P}=0.014)$ and lymph nodes metastasis $(\mathrm{P}<0.01)$. However, the relative MALAT1 expression level was not associated with other parameters such as gender $(P=0.651)$, age $(P=0.984)$, nor tumor differentiation $(P=0.991)$.

\section{Knock down of MALAT1 in ESCC cells decreased cell growth in vitro and in vivo}

Due to the abnormal expression of MALAT1 in ESCC cell lines and tissues, we speculated that it might have some potential roles in ESCC progression. To assess the role of MALAT1 in ESCC growth, we first silenced MALAT1 expression in EC109 and EC9706 by small interfering RNA. The siRNA which decreased MALAT1 expression level by more than $70 \%$ was chosen for further experiments. Of these two siRNAs, siRNA1 was chosen for further assays of EC109 while siRNA2 was selected for EC9706 (Figure 2A).

CCK8 assays revealed that cell growth was suppressed in both cell lines transfected with siRNAs compared with negative control (Figure 2B), colony-formation ability was also reduced by MALAT1 silencing in EC9706 and EC109 cells (Figure 2C). The inhibitory effect of MALAT1 knockdown on ESCC proliferation was also observed in a nude mice tumor growth model. Growth of tumors from the MALAT1-depleted xenografts was significantly inhibited compared with the control group (Figure 2D and E).

\section{Knockdown of MALAT1 impaired cell migration and invasion}

We investigated cancer cells migratory and invasive capacity through transwell assays. Compared with control groups, MALAT1-deficient cells displayed impeded migration in both EC109 and EC9706 cells. The migration decreased by approximately $38.2 \%$ and $25.6 \%$ respectively (Figure 3A). Similarly, invasion of EC109 and EC9706 cells was reduced by $34.2 \%$ and $39.8 \%$ separately following silencing of MALAT1 (Figure 3B).

\section{Knockdown of MALAT1 induced cell cycle arrest by activation of the ATM-CHK2 pathway}

Given that MALAT1 obviously promotes ESCC cell growth in vitro and in vivo, we next determined whether MALAT1 has any impact on cell cycle and apoptosis. In both cell lines, a significantly increased proportion of G2/ $M$ phase cells were observed among MALAT1 knockdown cells compared with that of control groups (Figure 4A). The apoptotic rate of EC109 and EC9706 cells transfected with siRNAs was also elevated (Figure 4B).

Further exploration of the possible mechanisms involved in the cell cycle arrest was performed by Westernblotting. For G2/M arrest is usually induced by increased DNA damage or genome instability, we proposed that the pathway responding to chromosome lesions may be activated by MALAT1 depletion. The results showed that the expression level of phosphorylated ATM, CHK2, CDC25C, and CDK1 were significantly increased upon MALAT1 depletion, while no detectable differences

Table 1 Correlation between MALAT1 expression and clinicopathological parameters of ESCC

\begin{tabular}{|c|c|c|c|c|}
\hline \multirow[b]{2}{*}{ Clinicopathological parameters } & & \multicolumn{3}{|l|}{ Expression pattern } \\
\hline & & Non-overexpression & Overexpression & $p$-value ${ }^{a}$ \\
\hline & & $n=29$ & $n=25$ & \\
\hline \multirow[t]{2}{*}{ Gender } & Male & 18 & 14 & 0.651 \\
\hline & Female & 11 & 11 & \\
\hline \multirow[t]{2}{*}{ Age } & $<60$ & 15 & 13 & 0.984 \\
\hline & $>60$ & 14 & 12 & \\
\hline \multirow[t]{2}{*}{ Differentiation } & Well & 7 & 6 & 0.991 \\
\hline & Moderate/Poor & 22 & 19 & \\
\hline \multirow[t]{2}{*}{ Metastasis } & Negative & 21 & 9 & $0.007^{b}$ \\
\hline & Positive & 8 & 16 & \\
\hline \multirow[t]{2}{*}{ Maxium diameter } & $\leq 4.0 \mathrm{~cm}$ & 19 & 8 & $0.014^{b}$ \\
\hline & $>4.0 \mathrm{~cm}$ & 10 & 17 & \\
\hline
\end{tabular}

\footnotetext{
${ }^{\mathrm{a}}$ Chi-squared test results.
}

${ }^{\mathrm{b}}$ Significant difference. 


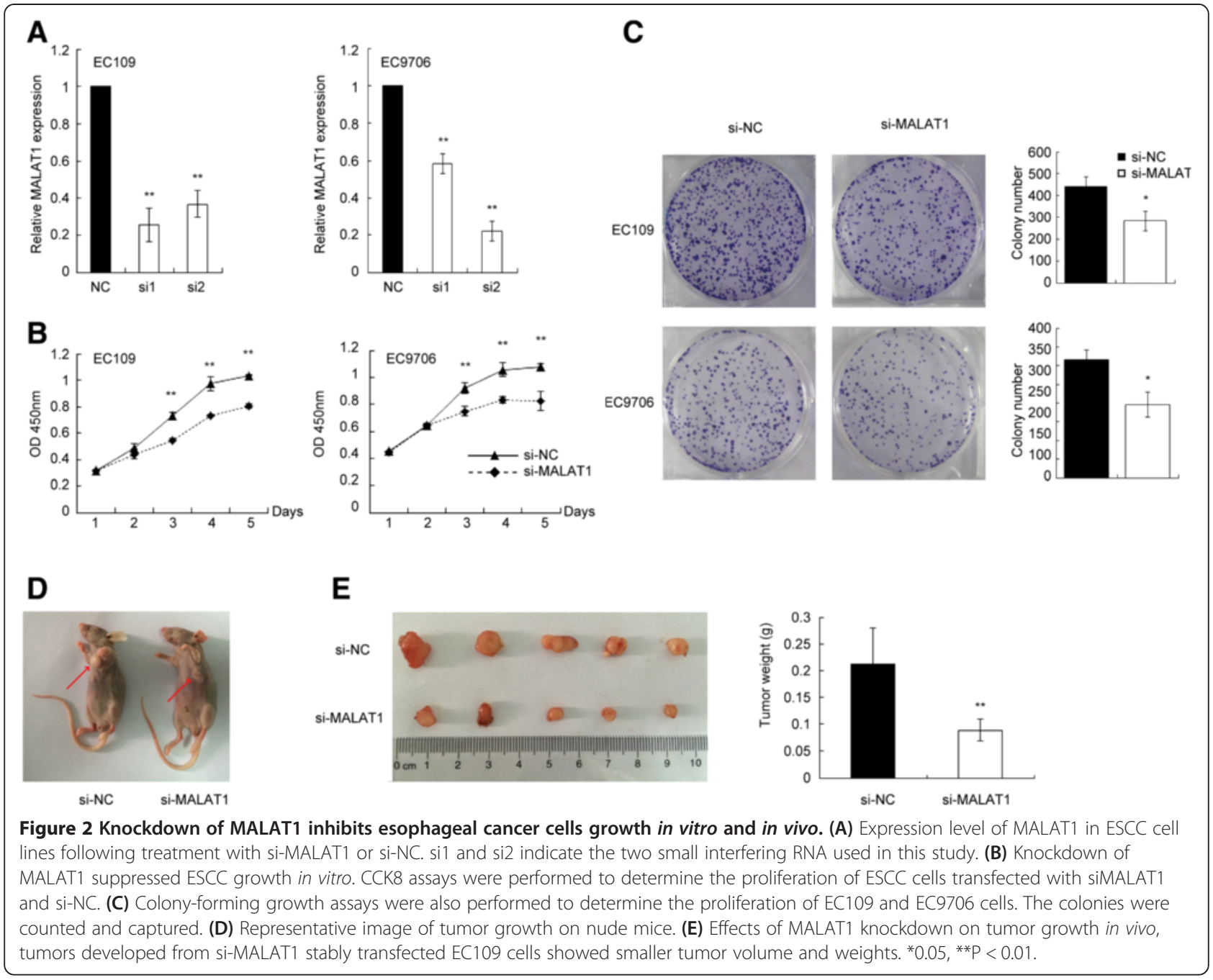

were observed for total ATM, CHK2, CDC25C, and CDK1 (Figure 4C). These data suggested that inhibition of MALAT1 may activate the ATM-CHK2 pathway, and eventually lead to G2/M arrest.

Then we detected the phosphorylated forms of ATM and CHK2 in five pairs of tissues, and the results showed that the phosphorylaion of ATM and CHK2 is increased in tumor tissues (Figure 5A), suggesting that the DNA damage events may actually occur in ESCC tissues, which can activate the ATM-CHK2 pathway and prevent tumor cells from growing too quickly.

To further confirm the association between MALAT1 and the ATM-CHK2 pathway, we examined the phosphorylated forms of ATM, CHK2, CDC25C, and CDK1 in ten ESCC tissues. Samples with high MALAT1 level (> mean value, and over-expressed in ESCC) showed relatively lower levels of ATM-CHK2 pathway phosphorylation (Figure $5 \mathrm{~B}$ ), suggesting a negative correlation between MALAT1 expression and phosphorylation of the ATM-CHK2 pathway in ESCC tissues, which is consistent with results from cell lines. Together these data suggested that high expression of MALAT1 promotes ESCC proliferation by dephosphorylation of the ATM-CHK2 pathway, which may release cells from $\mathrm{G} 2 / \mathrm{M}$ arrest, resulting in uncontrolled cell cycle and tumor growth.

\section{Amplification of MALAT1 in tumor tissues may partially contribute to its over-expression}

To explore the underlying mechanisms contributing to MALAT1 up-regulation in ESCC tissues, we first investigated the methylation status of the CpG island at the MALAT1 promoter in ten paired tissues by bisulfite sequencing. We sequenced the site of the $269 \mathrm{bp}-430 \mathrm{bp}$ of the CpG island, and the results revealed the total hypomethylation rate in both tumor and normal tissues, with the average frequency of methylation merely $4.4 \%$ in normal tissues and $4.7 \%$ in ESCC tissues (Figure 6A and B). We then treated EC109 and EC9706 cells with DNA demethylase agent (5-aza-CdR), and the results showed that $\mathrm{CDH} 1$ was up-regulated in agent treated 


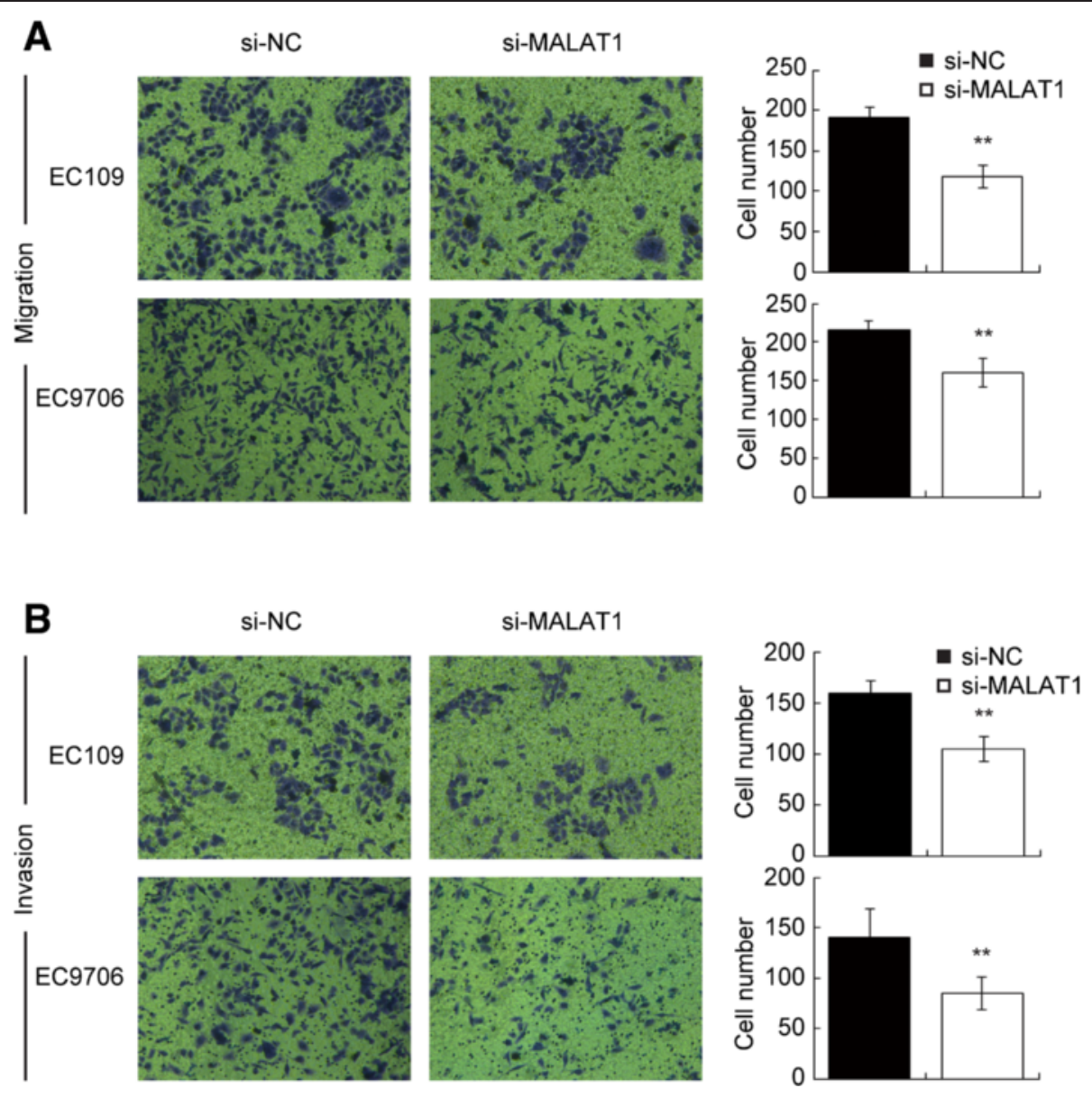

Figure 3 Inhibition of MALAT1 impaired invasive and migratory capacity of esophageal cancer cells. Representative images of the transwell migration (A) and invasion (B) assays, the bar chart represented the number of cells migrated or invaded into the lower chambers; Magnification: $100 \times$. ${ }^{*} \mathrm{P}<0.05,{ }^{*} \mathrm{P}<0.01$.

ells, suggesting that demethylation by 5 -aza-CdR is effective. However, MALAT1 expression was not significantly changed in demethylase treated cells (Figure 6C). These data supported the idea that there were no obvious effects of CpG island methylation status on MALAT1 expression.

We next detected the copy number of MALAT1 in the 54 tumor tissues by AccuCopy ${ }^{\mathrm{Tm}}$, for MALAT1 is located on human chromosome 11q13, which is reported to be frequently amplified in ESCC tissues. Amplification of MALAT1 was found in 12 tumor tissues (Figure 6D), among which nine showed increased MALAT1 expression over their counterparts (Figure 6E), while lack of over-expression in tumor tissues without amplification was observed in $48.1 \%$ of the cases, suggesting significant correlation between MALAT1 over-expression and its amplification (Table 2). These results indicated that up-regulation of MALAT1 observed in ESCC tissues might be partially due to amplification of the MALAT1 genomic region.
No association of germline copy number variation with somatic copy number amplification or ESCC risk was found in the case-control study

According to Database of Genomic Variation, both germline copy number gains and losses within the MALAT1 locus were recurrently identified by multiple research groups (Additional file 4: Figure S1A). Here we detected whether the germline copy number variation of MALAT1 may lead to tissue copy number amplification and is associated with ESCC risk in a case-control study, which contained blood samples from the 54 patients mentioned above. The probe for MALAT1 copy number detection was designed to overlap with the recorded CNV Variation_65973 within the MALAT1 genominc region (Additional file 4: Figure S1A). Finally we found no germline copy number gain in whole samples while copy number loss in twelve patients and eight controls (Additional file 4: Figure S1B), suggesting that the amplification in tissues may not 


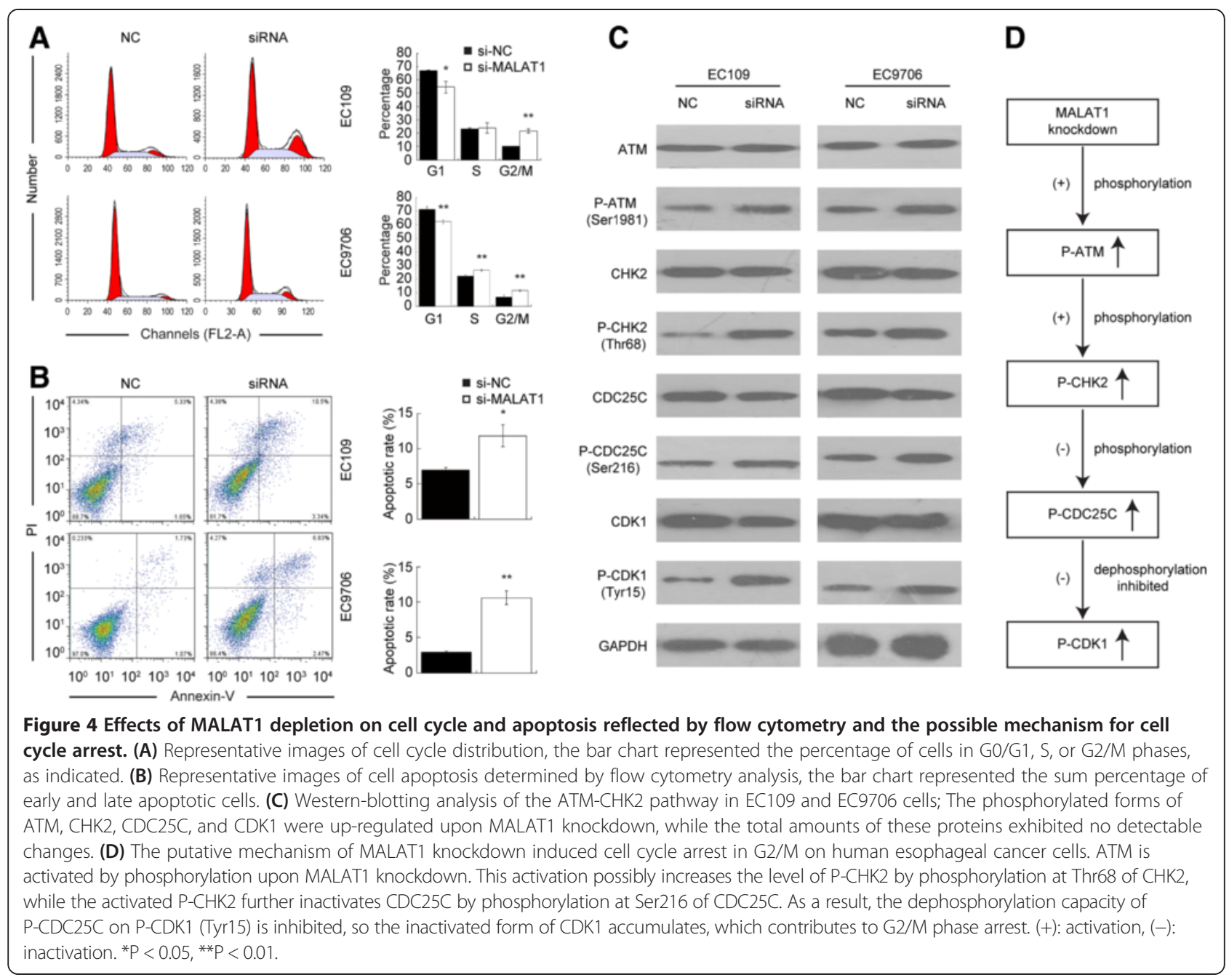

come from germline source, and the germline $\mathrm{CNV}$ should not be a risk indicator for ESCC (Table 3).

\section{Discussion}

Roles of lncRNAs in carcinogenesis have attracted more attention than ever before, as recent studies have found that IncRNAs are closely associated with tumor growth, epithelial-mesenchymal transition, and metastasis. However, little is known about their roles in ESCC. Previously researchers have discovered that the IncRNAs HOTAIR, POU3F3 and SPRY4-IT1 promoted ESCC progression and may be used as prognostic biomarkers, suggesting the critical roles of lncRNAs in ESCC [26-28].

Among these emerging functional stars, MALAT1 has been widely accepted as a key regulator in development and carcinogenesis, which participates in biological events through various mechanisms, such as chromosome modification [13], mediating mRNA relocation [29], and alternative splicing $[15,17,30]$. Recent studies have confirmed its oncogenic role in multiple cancer types, such as bladder, breast, and prostate cancers [31-33]. However, no studies on how it functions in human ESCC have been performed until now. Here we characterized its roles in ESCC for the first time.

First, we measured the expression level of MALAT1 in cell lines and clinical tissues by qRT-PCR. Overexpression of MALAT1 was found in 46.3\% of ESCC tissues, mostly in advanced tumor tissues with larger tumor size and lymph nodes metastasis, indicating that MALAT1 may primarily participate in ESCC advancing but not initialization, which may be a promising biomarker for late-stage ESCC with metastasis. Inhibition of MALAT1 suppressed tumor growth in vitro and in vivo, as well as cell migratory and invasive capacity, confirming its oncogenic roles in ESCC.

Further results suggested that knockdown of MALAT1 inhibited ESCC cell proliferation by inducing G2/M stage arrest and increasing the apoptosis ratio. The role of MALAT1 in G2/M transition regulation has been previously recognized, but the underlying mechanisms remain largely elusive. Yang et al. hypothesized that MALAT1 interacted with nuclear protein hnRNP $\mathrm{C}$ and facilitated 


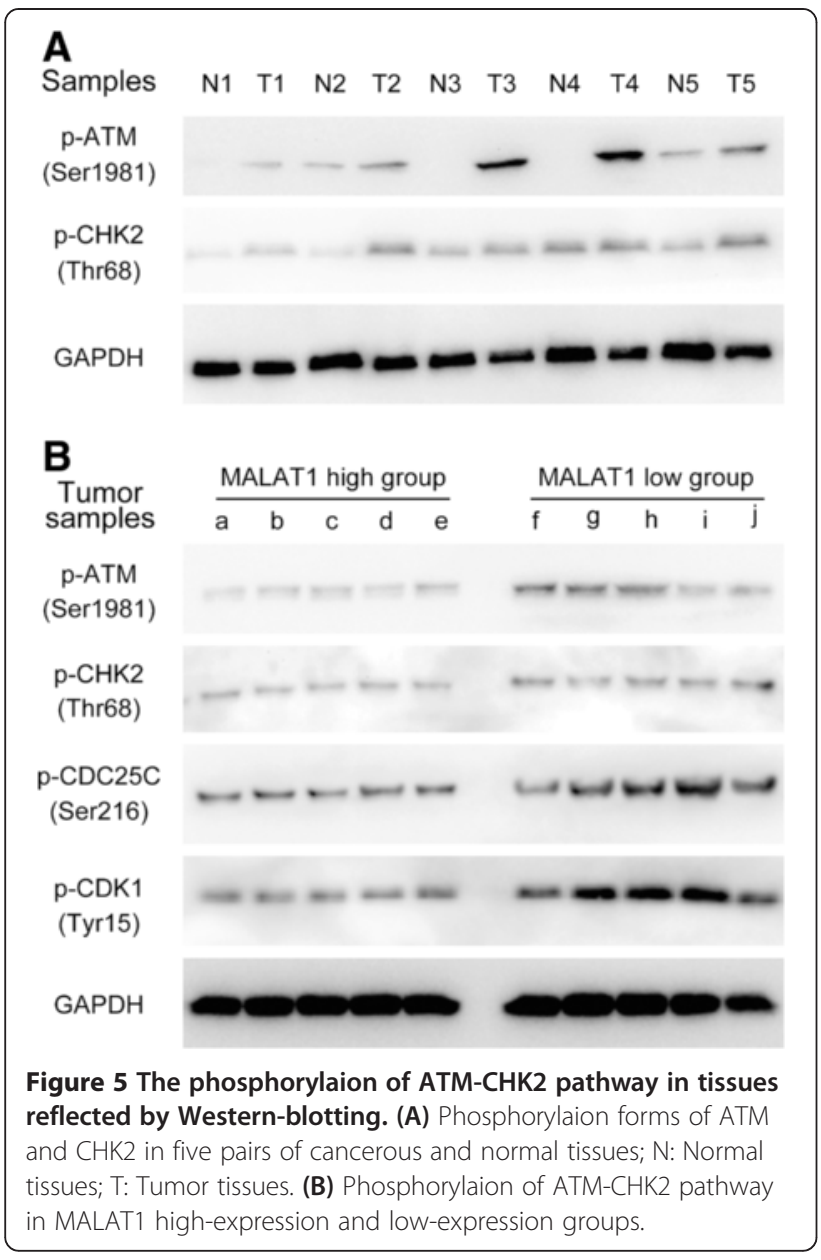

its cytoplasmic translocation in the G2/M phase [18], thereby regulating the progression of the cell cycle through hnRNP C. Meanwhile, Tripathi et al. reported that MALAT1-depleted cells displayed reduced expression of $\mathrm{B}-\mathrm{MYB}$, an oncogenic transcriptional factor involved in G2/M progression, resulting in cell cycle arrest [17]. Tripathi et al. also found increased DNA damage occurred in MALAT1 depleted cells, which should be a triggering signal for $\mathrm{G} 2 / \mathrm{M}$ arrest. DNA damage is a common event which occurs in tumor progression, induced by both the tumor microenvironment and genomic instability. The ATM-CHK2 pathway is an important component of cell cycle regulation, which responds to DNA damage signals, and prevents tumors growing too fast by arresting cell cycle in G2/M stage [34]. Here we found inactivation of ATM-CHK2 pathway by MALAT1 over-expression for the first time.

Results from Western-blotting showed that the phosphorylated forms of ATM, CHK2, CDC25C, and CDK1 were upregulated upon MALAT1 knockdown. As we know, ATM is a key regulator in cell cycle moderating, which may be phosphorylated at Ser1981 in response to environmental disturbance and genome instability, such as toxicant-induced or spontaneous DNA damage [35-37]. The phosphorylated form of ATM could subsequently activate $\mathrm{CHK} 2$ by phosphorylating $\mathrm{CHK} 2$ at the Thr68 site [38], and the activated CHK2 played its role in suppressing CDC25C by phosphorylation on Ser216 of CDC25C [39]. The CDC25C was a phosphatase which can direct dephosphorylation of P-CDK1 (Thr14, Tyr15) and triggers entry into mitosis. When phosphorylated, the P-CDC25C showed a reduced ability to dephosphorylate P-CDK1, which was reported to be an inactive form of CDK1, preventing cell cycle progression [40]. As a result, P-CDK1 accumulation occurred and the cell cycle was arrested in G2/M (Figure 4D).

Results from ESCC tissues further supported the idea that MALAT1 promotes tumor growth by inactivating the cell cycle checkpoint. In ESCC tissues, MALAT1 expression was negatively associated with phosphorylation of the ATM-CHK2 pathway, suggesting over-expression of MALAT1 may contribute to rapid tumor growth by suppressing cell cycle arrest. In total, our results highlighted the importance of MALAT1 in ATM-CHK2 pathway regulation, which may help us to interpret the mechanisms on how MALAT1 regulates tumor growth from a new perspective. However, more detailed information about the association between MALAT1 expression and ATM-CHK2 pathway is still required.

Although over-expression of MALAT1 has been reported in multiple cancer types, little is known about the factors which contribute to its up-regulation. Here we sought to explore the uncharted factors contributing to its up-regulation. Methylation of the $\mathrm{CpG}$ island in the promoter may be a crucial mechanism in regulating gene expression on an epigenetic level [41]. By computationally screening the promoter of MALAT1, we found a CpG island which is $441 \mathrm{bp}$ long. As no previous studies revealed whether this $\mathrm{CpG}$ island was associated with MALAT1 expression, we checked the correlation between the methylation status of the $\mathrm{CpG}$ island and MALAT1 expression level by bisulfite sequencing. The total methylation rate in both groups were extremely low and showed no apparent differences, indicating that the methylation status of MALAT1 promoter may not be a major factor contributing to its deregulation. No obvious changes in MALAT1 expression level upon treatment of ESCC cells with demethylase 5-aza-cdR further supported this idea.

MALAT1 is located on 11q13 of the human genome, which has been verified to be frequently amplified in ESCC tissues [42,43], and recent studies have discovered the tight correlation between lncRNAs amplification with their expression level $[44,45]$, we then detected the genomic copy number of MALAT1 in the 54 tumor tissues mentioned above by a newly developed method AccuCopy ${ }^{\mathrm{Tm}}$, which has been applied in other excellent 

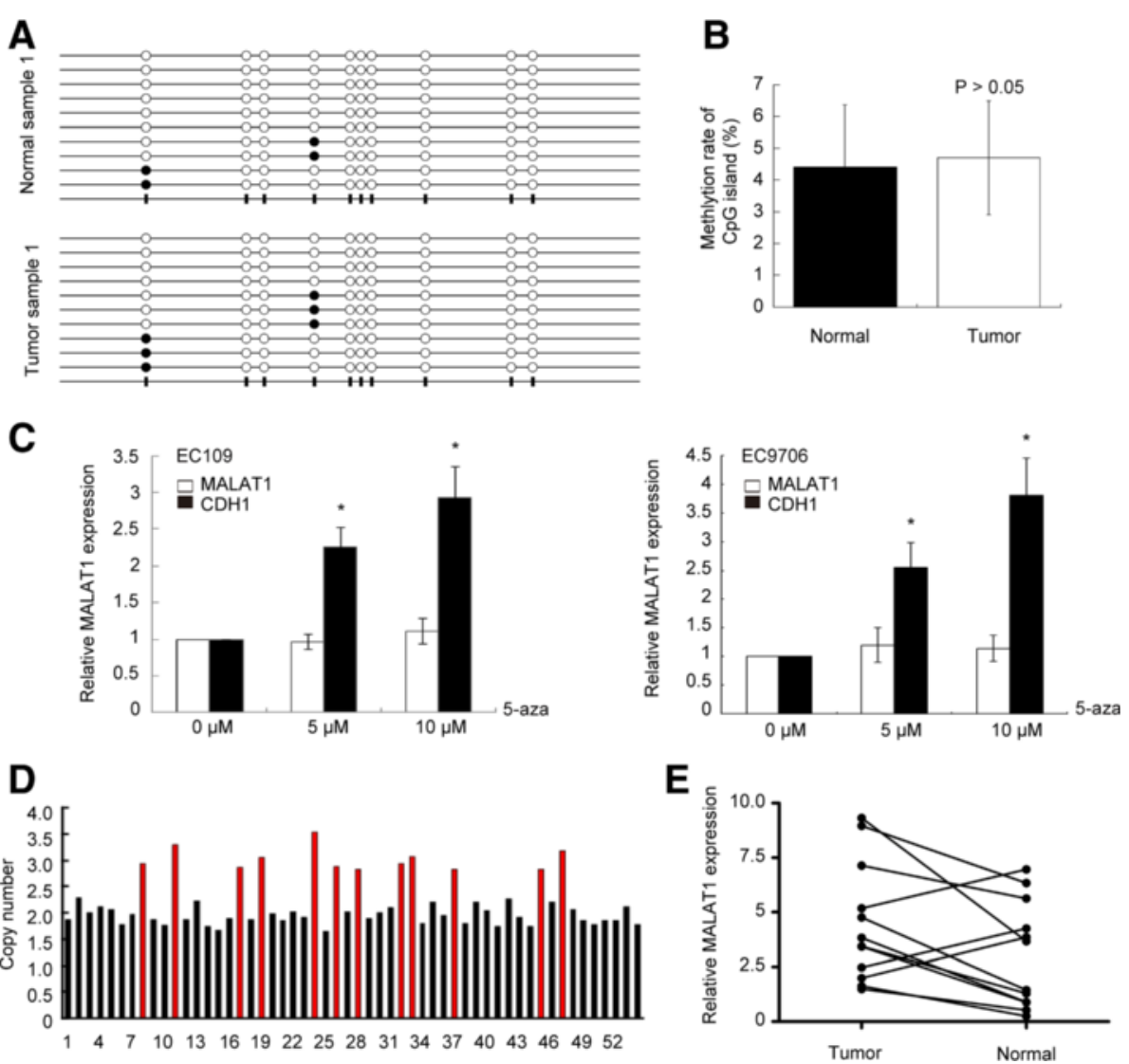

Figure 6 Effects of CpG island methylation status and copy number amplification on MALAT1 expression level. (A) Methylation status of the CPG island in a representative pair of clinical tissues, assessed by bisulfite sequencing. Open and filled squares denote un-methylated and methylated CpG sites respectively; each row represents a single clone. (B) No obvious difference in CpG island methylation frequency was observed in the ten pairs of tissues. A paired Student's t-test was performed to determine the statistical significance. (C) The expression level of MALAT1 and CDH1 in EC109 and EC9706 cells following treatment with demethylase agent 5-aza-cdR (0, 5, and $10 \mu \mathrm{M})$. (D) Genomic copy number of MALAT1 in tumor tissues calculated by AccuCopy ${ }^{\text {TM }}$ system. Red bars represented samples with MALAT1 amplification in tumor tissues. (E) Relative expression level of MALAT1 (normalized to GAPDH) in the twelve pairs of tissues, which showed gene amplification in tumor tissues. ${ }^{*} \mathrm{P}<0.05,{ }^{*} \mathrm{P}<0.01$.

works, and proven to be efficient and accurate $[25,46]$. The results showed that amplification of MALAT1 was found in 12 ESCC tissues, among which nine showed an overexpression pattern, implicating that the up-regulation of MALAT1 in tumor tissues may partially be attributed to copy number amplification in somatic tissues.

As amplification of MALAT1 was found in ESCC tissues, then we wondered how the amplification in tumor tissues occurred. Previous studies have proclaimed that the germline copy number variation may be an important source of somatic genome abnormality [46,47], and germline copy number gains across MALAT1 have been reported by other groups $[48,49]$. We therefore tested if this association also existed in the MALAT1 region. In this study, we genotyped the copy number of MALAT1 in 201 ESCC patients (the 54 patients mentioned above included) and 193 healthy controls. Surprisingly, we found no copy number gain in germline samples, indicating that the CNV status may differ in diverse ethnic groups, and it is hard to predict whether the amplification of MALAT1 in

Table 2 Association between MALAT1 amplification and its over-expression in ESCC tissues

\begin{tabular}{|c|c|c|c|c|}
\hline & & \multicolumn{2}{|l|}{ Expression level } & \multirow[t]{2}{*}{$p$-value } \\
\hline & & Over-expression & Non over-expression & \\
\hline \multirow[t]{2}{*}{ Copy number status in tumor tissues } & Amplification & 9 & 3 & $0.046^{\mathbf{b}}$ \\
\hline & Non-amplification & 16 & 26 & \\
\hline
\end{tabular}


Table 3 No association of germline copy number variation with ESCC risk was found in the case-control study

\begin{tabular}{lllllll}
\hline genotypes & No. of cases & No. of controls & Crude OR $(\mathbf{9 5} \% \mathbf{C l})$ & $\boldsymbol{p}$-value & Adjusted OR (95\%Cl) & $\boldsymbol{p}$-value \\
\hline$+/+^{\mathbf{a}}$ & 189 & 185 & 1.00 (reference) & & 1.00 (reference) \\
$+/-^{\mathbf{a}}$ & 12 & 8 & $1.437(0.574-3.596)$ & 0.438 & $1.385(0.544-3.522)$ & 0.494
\end{tabular}

aGenotypes: +/+, wild-type; +/-, hemizygous deletion.

${ }^{\mathrm{b}}$ Adjusted by gender, age and smoking status.

tumor tissues came from the germline origin. So we believe that the amplification of MALAT1 in ESCC tissues may be a complicated event arising during the progression phase of ESCC, but not being simply derived from germline origin. Moreover, we did not find any frequency discrepancy of germline copy number loss between the cases and controls groups, indicating that the germline copy number variation on MALAT1 should not be treated as an ESCC susceptibility indicator, in spite of its significant role in ESCC.

\section{Conclusions}

Together these findings indicate that MALAT1 is a player of great importance in ESCC. It may promote ESCC growth by moderating the ATM-CHK2 pathway which is involved in cell cycle orchestrating, and the amplification of MALAT1 may be an important cause for its up-regulation in ESCC tissues. However, much more work is still required to determine the detailed mechanisms it functions in ESCC and the potentiality of MALAT1 as a therapeutic target for ESCC.

\section{Additional files}

Additional file 1: Table S1. Distribution of demographic characteristics for cases and controls.

Additional file 2: Table S2. Primers used in this study.

Additional file 3: Table S3. Sequences of the small interfering RNAs and negative control.

Additional file 4: Figure S1. No difference in copy number variation between the cases and controls groups was found. (A) Copy number variations across MALAT1 were identified by multiple groups; Red: Copy number losses; Blue: copy number gains. (B) Copy numbers of MALAT1 in the case-control population calculated by AccuCopy ${ }^{\mathrm{TM}}$. Each bar represents the copy number for its carrier; CA: cases; CTR: controls.

\begin{abstract}
Abbreviations
ESCC: Esophageal squamous cell carcinoma; NSCLC: Non-small cell lung cancer; CNV: Copy number variation; IncRNA: Long noncoding RNA; CDNA: Complementary DNA; qRT-PCR: Quantitative Reverse Transcription-PCR; HOTAIR: HOX Transcript Antisense RNA; MEG3: Maternally expressed 3; GAS5: Growth arrest-specific 5; MALAT1: Metastasis associated lung adenocarcinoma transcript 1; LB: Lysogeny broth; X-gal: 5-Bromo-4chloro-3-indolyl $\beta$-D-galactopyranoside; IPTG: Isopropyl $\beta$-D-1-Thiogalactopyranoside; ATM: Ataxia telangiectasia-mutated kinase; $\mathrm{CHK}$ 2: Checkpoint kinase 2; CDC25C: Cell division cycle 25C; CDK1: Cyclin-dependent kinase 1; $\mathrm{CDH1}$ : Cadherin 1; GAPDH: Glyceraldehyde-3-phosphate dehydrogenase.
\end{abstract}

\section{Competing interest}

The authors declare that they have no competing interests.

\section{Authors' contributions}

LWH designed the experiments, performed the main experimental work, and drafted the manuscript. YYW and DLT performed real-time PCR, Western blotting, and statistical analysis. HM and KW participated in the cell culture and manuscript preparation. $Y B$ and $K Y$ conceived of the study, and participated in design, coordination and supervision. All authors have read and approved the final manuscript.

\section{Acknowledgements}

This work was supported by grants from National Natural Science Foundation of China [No. 81172379$]$. We thanked Xinyue Yao, Limeng Dai and Xuedan Chen for writing assistances and Xingying Guan for clinical samples collection.

Received: 2 November 2014 Accepted: 4 January 2015

Published online: 22 January 2015

\section{References}

1. Wang B, Yin BL, He B, Chen C, Zhao M, Zhang W, et al. Overexpression of DNA damage-induced 45 alpha gene contributes to esophageal squamous cell cancer by promoter hypomethylation. J Exp Clin Cancer Res. 2012;31:11.

2. Demeester SR. Epidemiology and biology of esophageal cancer. Gastrointest Cancer Res. 2009;3:S2-5.

3. Song H, Xu B, Yi J. Clinical significance of stanniocalcin-1 detected in peripheral blood and bone marrow of esophageal squamous cell carcinoma patients. J Exp Clin Cancer Res. 2012;31:35.

4. Wahlestedt C. Targeting long non-coding RNA to therapeutically upregulate gene expression. Nat Rev Drug Discov. 2013;12:433-46.

5. Rinn JL, Kertesz M, Wang JK, Squazzo SL, Xu X, Brugmann SA, et al. Functional demarcation of active and silent chromatin domains in human HOX loci by noncoding RNAs. Cell. 2007;129:1311-23.

6. Dimond A, Fraser P. Molecular biology. Long noncoding RNAs Xist in three dimensions. Science. 2013;341:720-1.

7. Denzler R, Agarwal V, Stefano J, Bartel DP, Stoffel M. Assessing the ceRNA hypothesis with quantitative measurements of miRNA and target abundance. Mol Cell. 2014;54:766-76.

8. Shi X, Sun M, Liu H, Yao Y, Song Y. Long non-coding RNAs: a new frontier in the study of human diseases. Cancer Lett. 2013;339:159-66.

9. Ji P, Diederichs S, Wang W, Boing S, Metzger R, Schneider PM, et al. MALAT-1, a novel noncoding RNA, and thymosin beta4 predict metastasis and survival in early-stage non-small cell lung cancer. Oncogene. 2003;22:8031-41.

10. Zheng HT, Shi DB, Wang YW, Li XX, Xu Y, Tripathi P, et al. High expression of InCRNA MALAT1 suggests a biomarker of poor prognosis in colorectal cancer. Int J Clin Exp Pathol. 2014;7:3174-81.

11. Gutschner T, Hammerle M, Eissmann M, Hsu J, Kim Y, Hung G, et al. The noncoding RNA MALAT1 is a critical regulator of the metastasis phenotype of lung cancer cells. Cancer Res. 2013;73:1180-9.

12. Han Y, Liu Y, Nie L, Gui Y, Cai Z. Inducing cell proliferation inhibition, apoptosis, and motility reduction by silencing long noncoding ribonucleic acid metastasis-associated lung adenocarcinoma transcript 1 in urothelial carcinoma of the bladder. Urology. 2013;81:209. e201-207.

13. Wang J, Su L, Chen X, Li P, Cai Q, Yu B, et al. MALAT1 promotes cell proliferation in gastric cancer by recruiting SF2/ASF. Biomed Pharmacother. 2014;68:557-64.

14. West JA, Davis CP, Sunwoo H, Simon MD, Sadreyev Rl, Wang Pl, et al. The long noncoding RNAs NEAT1 and MALAT1 bind active chromatin sites. Mol Cell. 2014:55:791-802.

15. Tripathi V, Ellis JD, Shen Z, Song DY, Pan Q, Watt AT, et al. The nuclear-retained noncoding RNA MALAT1 regulates alternative splicing by modulating SR splicing factor phosphorylation. Mol Cell. 2010;39:925-38.

16. Kuo IY, Wu CC, Chang JM, Huang YL, Lin CH, Yan JJ, et al. Low SOX17 expression is a prognostic factor and drives transcriptional dysregulation and esophageal cancer progression. Int J Cancer. 2014;135:563-73.

17. Tripathi V, Shen Z, Chakraborty A, Giri S, Freier SM, Wu X, et al. Long noncoding RNA MALAT1 controls cell cycle progression by regulating the 
expression of oncogenic transcription factor B-MYB. PLoS Genet. 2013;9: e1003368.

18. Yang F, Yi F, Han X, Du Q, Liang Z. MALAT-1 interacts with hnRNP C in cell cycle regulation. FEBS Lett. 2013;587:3175-81.

19. Tee $A E$, Ling D, Nelson C, Atmadibrata B, Dinger ME, Xu N, et al. The histone demethylase JMJD1A induces cell migration and invasion by up-regulating the expression of the long noncoding RNA MALAT1. Oncotarget. 2014:5:1793-804

20. Fan Y, Shen B, Tan M, Mu X, Qin Y, Zhang F, et al. TGF-beta-induced upregulation of malat1 promotes bladder cancer metastasis by associating with suz12. Clin Cancer Res. 2014;20:1531-41.

21. Han Y, Liu Y, Zhang H, Wang T, Diao R, Jiang Z, et al. Hsa-miR-125b suppresses bladder cancer development by down-regulating oncogene SIRT7 and oncogenic long non-coding RNA MALAT1. FEBS Lett. 2013;587:3875-82.

22. Leucci E, Patella F, Waage J, Holmstrom K, Lindow M, Porse B, et al. microRNA-9 targets the long non-coding RNA MALAT1 for degradation in the nucleus. Sci Rep. 2013;3:2535.

23. Lee EJ, Lee BB, Han J, Cho EY, Shim YM, Park J, et al. CpG island hypermethylation of E-cadherin $(\mathrm{CDH} 1)$ and integrin alpha4 is associated with recurrence of early stage esophageal squamous cell carcinoma. Int J Cancer. 2008:123:2073-9.

24. Ling ZQ, Li P, Ge MH, Zhao X, Hu FJ, Fang XH, et al. Hypermethylationmodulated down-regulation of $\mathrm{CDH} 1$ expression contributes to the progression of esophageal cancer. Int J Mol Med. 2011;27:625-35.

25. Du R, Lu C, Jiang Z, Li S, Ma R, An H, et al. Efficient typing of copy number variations in a segmental duplication-mediated rearrangement hotspot using multiplex competitive amplification. J Hum Genet. 2012;57:545-51.

26. Li W, Zheng J, Deng J, You Y, Wu H, Li N, et al. Increased levels of the long intergenic non-protein coding RNA POU3F3 promote DNA methylation in esophageal squamous cell carcinoma cells. Gastroenterology. 2014;146:1714-26. e1715.

27. Li X, Wu Z, Mei Q, Guo M, Fu X, Han W. Long non-coding RNA HOTAIR, a driver of malignancy, predicts negative prognosis and exhibits oncogenic activity in oesophageal squamous cell carcinoma. Br J Cancer. 2013;109:2266-78.

28. Xie HW, Wu QQ, Zhu B, Chen FJ, Ji L, Li SQ, et al. Long noncoding RNA SPRY4-IT1 is upregulated in esophageal squamous cell carcinoma and associated with poor prognosis. Tumour Biol. 2014:35:7743-54.

29. Yang L, Lin C, Liu W, Zhang J, Ohgi KA, Grinstein JD, et al. ncRNA- and PC2 methylation-dependent gene relocation between nuclear structures mediates gene activation programs. Cell. 2011;147:773-88.

30. Anko ML, Neugebauer KM. Long noncoding RNAs add another layer to pre-mRNA splicing regulation. Mol Cell. 2010;39:833-4.

31. Ying L, Chen Q, Wang Y, Zhou Z, Huang Y, Qiu F. Upregulated MALATcontributes to bladder cancer cell migration by inducing epithelial-tomesenchymal transition. Mol Biosyst. 2012;8:2289-94.

32. Zhao Z, Chen C, Liu Y, Wu C. 17beta-Estradiol treatment inhibits breast cell proliferation, migration and invasion by decreasing MALAT-1 RNA level. Biochem Biophys Res Commun. 2014;445:388-93.

33. Ren S, Liu Y, Xu W, Sun Y, Lu J, Wang F, et al. Long noncoding RNA MALAT-1 is a new potential therapeutic target for castration resistant prostate cancer. J Urol. 2013;190:2278-87.

34. Matsuoka S, Huang M, Elledge SJ. Linkage of ATM to cell cycle regulation by the Chk2 protein kinase. Science. 1998;282:1893-7.

35. Bakkenist CJ, Kastan MB. DNA damage activates ATM through intermolecular autophosphorylation and dimer dissociation. Nature. 2003:421:499-506.

36. Dodson GE, Shi Y, Tibbetts RS. DNA replication defects, spontaneous DNA damage, and ATM-dependent checkpoint activation in replication protein A-deficient cells. J Biol Chem. 2004:279:34010-4.

37. Iwahori S, Kohmon D, Kobayashi J, Tani Y, Yugawa T, Komatsu K, et al. ATM regulates $\mathrm{Cdt1}$ stability during the unperturbed $\mathrm{S}$ phase to prevent re-replication. Cell Cycle. 2014;13:471-81.

38. Ahn JY, Schwarz JK, Piwnica-Worms H, Canman CE. Threonine 68 phosphorylation by ataxia telangiectasia mutated is required for efficient activation of Chk2 in response to ionizing radiation. Cancer Res. 2000;60:5934-6.

39. Thanasoula M. Escandell JM, Suwaki N, Tarsounas M. ATM/ATR checkpoint activation downregulates $\mathrm{CDC} 25 \mathrm{C}$ to prevent mitotic entry with uncapped telomeres. EMBO J. 2012:31:3398-410.
40. Khammanivong A, Wang C, Sorenson BS, Ross KF, Herzberg MC. S100A8/A9 (calprotectin) negatively regulates $\mathrm{G} 2 / \mathrm{M}$ cell cycle progression and growth of squamous cell carcinoma. PLoS One. 2013;8:e69395.

41. Shiovitz S, Bertagnolli MM, Renfro LA, Nam E, Foster NR, Dzieciatkowski S, et al. CpG island methylator phenotype is associated with response to adjuvant irinotecan-based therapy for stage III colon cancer. Gastroenterology. 2014; 147:637-45

42. Shi ZZ, Shang L, Jiang YY, Hao JJ, Zhang Y, Zhang TT, et al. Consistent and differential genetic aberrations between esophageal dysplasia and squamous cell carcinoma detected by array comparative genomic hybridization. Clin Cancer Res. 2013;19:5867-78.

43. Ying J, Shan L, Li J, Zhong L, Xue L, Zhao H, et al. Genome-wide screening for genetic alterations in esophageal cancer by aCGH identifies 11q13 amplification oncogenes associated with nodal metastasis. PLoS One. 2012; :e39797.

44. Takahashi Y, Sawada G, Kurashige J, Uchi R, Matsumura T, Ueo H, et al. Amplification of PVT-1 is involved in poor prognosis via apoptosis inhibition in colorectal cancers. Br J Cancer. 2014;110:164-71.

45. Hu Y, Wang J, Qian J, Kong X, Tang J, Wang Y, et al. Long non-coding RNA GAPLINC regulates CD44-dependent cell invasiveness and associates with poor prognosis of gastric cancer. Cancer Res. 2014;74:6890-902.

46. Liu B, Yang L, Huang B, Cheng M, Wang H, Li Y, et al. A functional copy-number variation in MAPKAPK2 predicts risk and prognosis of lung cancer. Am J Hum Genet. 2012;91:384-90.

47. Huang L, Yu D, Wu C, Zhai K, Jiang G, Cao G, et al. Copy number variation at $6 q 13$ functions as a long-range regulator and is associated with pancreatic cancer risk. Carcinogenesis. 2012;33:94-100.

48. Conrad DF, Pinto D, Redon R, Feuk L, Gokcumen O, Zhang Y, et al. Origins and functional impact of copy number variation in the human genome. Nature. 2010;464:704-12.

49. Park H, Kim Jl, Ju YS, Gokcumen O, Mills RE, Kim S, et al. Discovery of common Asian copy number variants using integrated high-resolution array CGH and massively parallel DNA sequencing. Nat Genet. 2010;42:400-5.

\section{Submit your next manuscript to BioMed Central and take full advantage of:}

- Convenient online submission

- Thorough peer review

- No space constraints or color figure charges

- Immediate publication on acceptance

- Inclusion in PubMed, CAS, Scopus and Google Scholar

- Research which is freely available for redistribution 